\title{
Tensile Deformation of Al Thin Films Studied by In-situ TEM and Molecular Dynamics Simulations
}

\author{
$\underline{\text { Lucia Bajtošová }}^{1}$, Barbora Křivská ${ }^{1}$, Jozef Veselý $^{1}$, Miroslav Cieslar ${ }^{1}$, Jan Hanuš ${ }^{1}$, Petr Harcuba ${ }^{1}$ and \\ Jan Fikar ${ }^{2}$ \\ ${ }^{1}$ Faculty of Mathematics and Physics, Charles University Prague, Czech Republic. \\ ${ }^{2}$ Institute of Physics of Materials, Academy of Sciences of the Czech Republic Brno, Czech Republic.
}

Mechanical properties of nanocrystalline films with grain sizes smaller than $100 \mathrm{~nm}$ have recently been a subject of interest due to their wide applicability in micromechanical devices. Restricted dimensions of these films are a cause of properties deviating from the ones observed in bulk materials [1]. A combination of mechanical tests with direct observation of the material structure by in-situ transmission electron microscope (TEM) [2] can be combined with molecular dynamics (MD) simulation [3], a computer simulation method which allows prediction of individual atom motion from numerical integration of equations of motion.

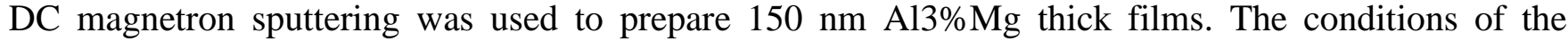
deposition were following: working pressure $0.34 \mathrm{~Pa}$, current $\sim 100 \mathrm{~mA}$ and voltage $\sim 5 \mathrm{~V}$. The film was sputtered on a glass substrate covered with polystyrene tape, which was then dissolved in toluene and acetone to obtain free standing samples. The film was in-situ annealed up to $640^{\circ} \mathrm{C}$ in TEM Jeol $2200 \mathrm{FS}$, operated at $200 \mathrm{kV}$. Dog-bone shaped specimen were prepared in scanning electron microscope Zeiss Auriga using focused ion beam (FIB) and fixed onto Hysitron Push-to-Pull (PTP) device with stiffness $150 \mathrm{~N} / \mathrm{m}$. The specimens were deformed in TEM Jeol 2200FS by Hysitron PI 95 TEM PicoIndenter equipped with a flat punch and characterized by Automated orientation phase mapping.

Automated orientation phase mapping of the annealed $150 \mathrm{~nm}$ thick Al film revealed polyhedral columnar grains with sizes ranging from 20 to $200 \mathrm{~nm}$. [110] texture in a direction perpendicular to the surface was observed. The samples were deformed in tension at strain rate $10^{-4} \mathrm{~s}^{-1}$ with the active area simultaneously captured on camera in BF TEM mode (Fig. 1). Extensive contrast changes in individual grains signifying dislocation activity (Fig. 1b - marked by arrows) in the grain boundraries (GB) or grain rotations were detected along with minor in-grain dislocation activity short before the crack formation. The crack propagation started at $9 \%$ strain and stress $700 \mathrm{MPa}$ and occurred along GB.

Large-Scale Atomic/Molecular Massively Parallel Simulator (LAMMPS) [4] was used to perform the MD simulations. A polycrystal structure in an orthogonal box with dimensions $x=200 \mathrm{~nm}, \mathrm{y}=200 \mathrm{~nm}, \mathrm{z}=200$ $\mathrm{nm}$ was created using Atomsk software [5]. Periodic boundary conditions were employed in $x$ and $y$ directions. Six columnar hexagonal grains with [110] texture in z direction were created and deformed at strain rate $2.10^{9} \mathrm{~s}^{-1}$ at $300 \mathrm{~K}$ up to $20 \%$ strain. Al MEAM potential was used to carry out the simulation. The simulation was repeated for the same polycrystal with modified size - thickness $\mathrm{z}=500 \mathrm{~nm}$ and width $\mathrm{x}=400$ and $\mathrm{y}=400 \mathrm{~nm}$. The results were visualized by Open Visualization Tool (OVITO) [6] with the common neighbor analysis (CNA) and dislocation analysis (DXA).

The results of the simulation are shown in Fig 2. At 3\% strain, one full dislocation was emitted from a GB, propagated through the grain (Fig 2. a,b) and was annihilated in the opposite GB. Aside from that, no in-grain dislocation activity was observed until $9 \%$ when two stacking fault planes bound by partial dislocations formed. Subsequently, tearing of the material along the GB perpendicular to the deformation 
direction started (Fig 2. d). Most of the deformation at strains under 9\% was carried out by a movement of dislocations inside GB. The deformation of polycrystal blocks with larges dimensions (200x200x500 $\mathrm{nm}^{3}$ and 400×400×200 $\mathrm{nm}^{3}$ ) yielded coincident results - dislocation movement inside GB, low in-grain dislocation activity and intergranular tearing at strains $\sim 10 \%$ and strain $4.5 \mathrm{GPa}$.

In conclusion, a relatively good compliance between the experimental observations and the simulation results was reached. Neither the change of sample thickness from 20 to $50 \mathrm{~nm}$, nor the change of grain size from 10 to $20 \mathrm{~nm}$ did significantly affect the simulation results. The tensile strength predicted in the simulation was several times higher than the experiment result [7].

\section{References:}

[1] A Tajik, H Jahed, InTech, p. 435.

[2] KS Kumar et al, Acta Materialia 51(2) (2003), p. 387.

[3] FF Abraham, Advances in Physics 35(1) (1986), p. 1.

[4] S Plimpton, J. Comp. Phys. 117 (1995), p. 1.

[5] P Hirel, Computer Physics Communications 197 (2015), p. 212.

[6] A Stukowski, Mater. Sci. Eng. 18 (2010), p. 015012.

[7] Authors would like to acknowledge the support from Grant Agency of Charles University under the project 574120 .
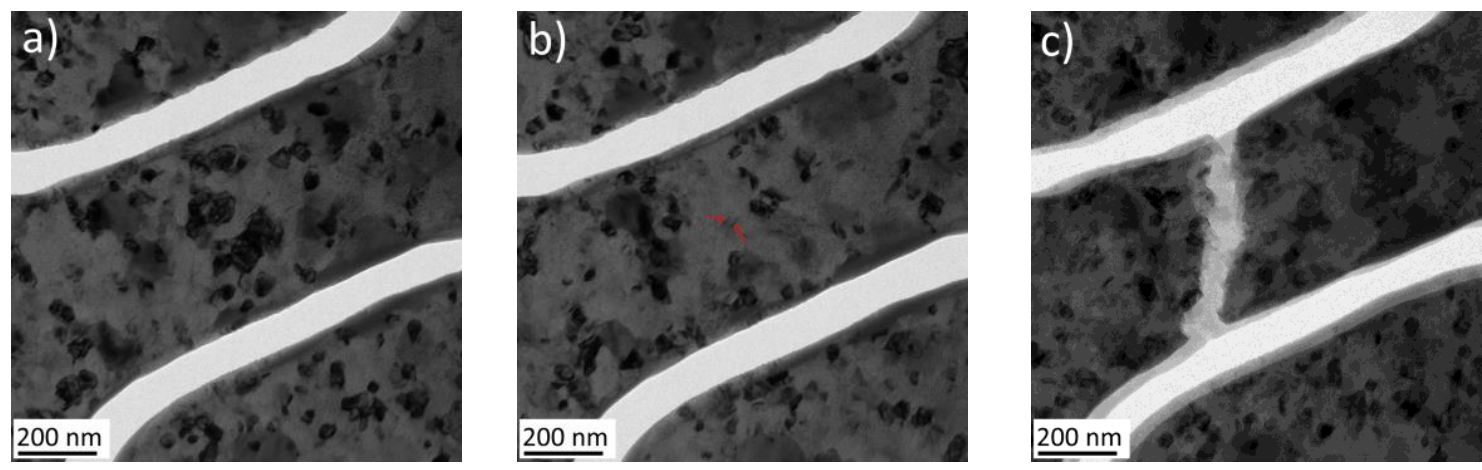

Figure 1. TEM images from in-situ tensile deformation experiment a) 0\%, b) 6\%, c) after rupture.
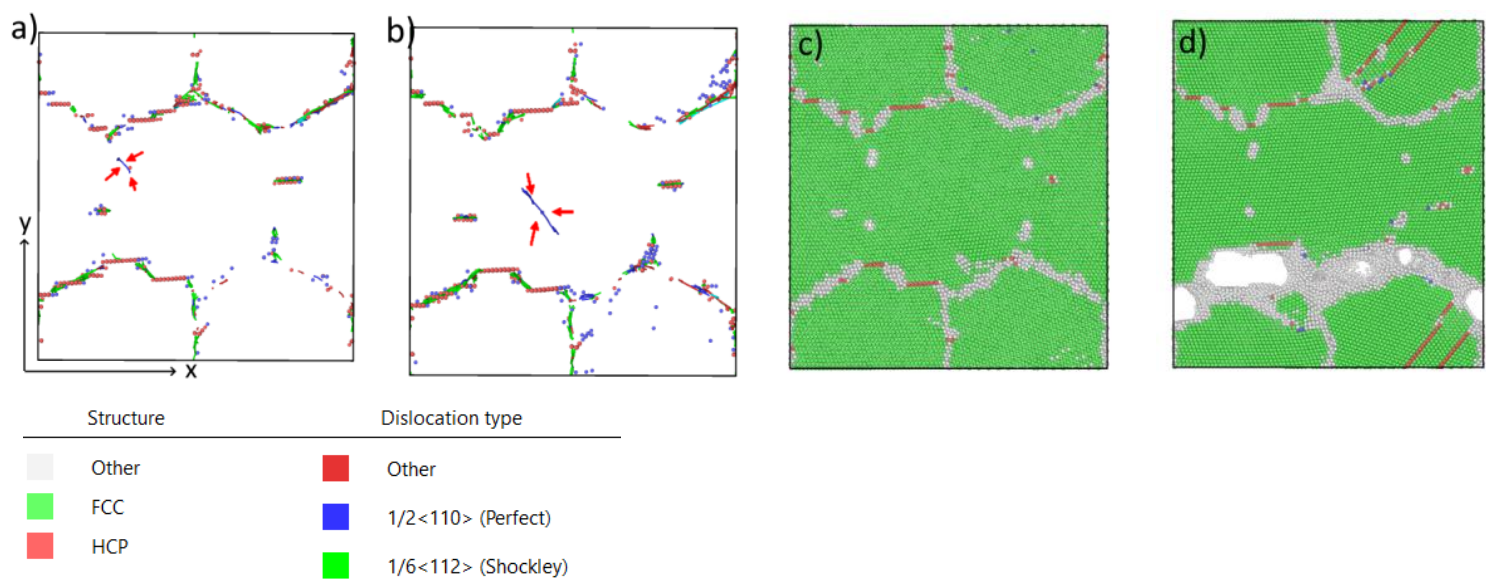

Figure 2. Visualization of MD simulation a) DXA 3\%, b) DXA 6\%, c) CNA 7\%, d) CNA $11 \%$ strain. 